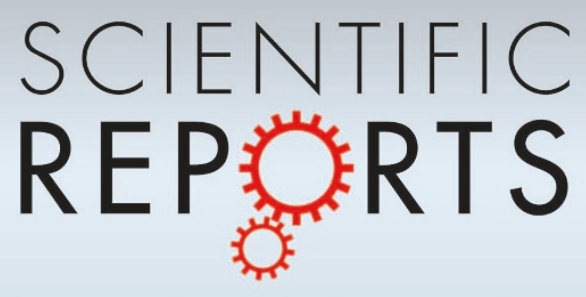

OPEN

SUBJECT AREAS:

NANOFABRICATION AND NANOPATTERNING

CELL DELIVERY

NANOPORES

MAGNETIC PROPERTIES AND MATERIALS

Received

1 April 2014

Accepted

7 August 2014

Published

15 September 2014

Correspondence and requests for materials should be addressed to

K.Y. Yyasuda.bmi@ tmd.ac.jp)

\section{Cup-Shaped Superparamagnetic Hemispheres for Size-Selective Cell Filtration}

\author{
Hyonchol Kim', Hideyuki Terazono ${ }^{1,2}$, Hiroyuki Takei ${ }^{1,3}$ \& Kenji Yasuda ${ }^{1,2}$
}

'Kanagawa Academy of Science and Technology, KSP East 31 10, 3-2-1 Sakado, Takatsu-ku, Kawasaki, Kanagawa $213-0012$, Japan, ${ }^{2}$ Department of Biomedical Information, Division of Biosystems, Institute of Biomaterials and Bioengineering, Tokyo Medical and Dental University, 2-3-10 Kanda-Surugadai, Chiyoda-ku, Tokyo 101-0062, Japan, ${ }^{3}$ Department of Life Sciences, Faculty of Life Sciences, Toyo University, 1-1-1 Izumino, Itakura-machi, Oura-gun, Gunma 374-0193, Japan.

We propose a new method of size separation of cells exploiting precisely size-controlled hemispherical superparamagnetic microparticles. A three-layered structure of a 2-nm nickel layer inserted between 15-nm silicon dioxide layers was formed on polystyrene cast spheres by vapor deposition. The polystyrene was then removed by burning and the hemispherical superparamagnetic microparticles, "magcups", were obtained. The standard target cells (CCRF-CEM, $12 \pm 2 \mu \mathrm{m}$ ) were mixed with a set of different sizes of the fabricated magcups, and we confirmed that the cells were captured in the magcups having cavities larger than $15 \mu \mathrm{m}$ in diameter, and then gathered by magnetic force. The collected cells were grown in a culture medium without any damage. The results suggest that this method is quick, simple and non-invasive size separation of target cells.

urification depending on size is one of the fundamental processes of cell purification especially when the specific molecular biomarker is not identified for the candidate cells. For example, differences in the size of cancer cells from that of normal blood cells were identified, and technologies for isolating such cancer cells from blood depending on their size were developed ${ }^{1-6}$. Membrane filtration is most widely used for such separation because of its preciseness and simple preparation, called ISET (isolation by size of epithelial tumor cells) ${ }^{1,2}$. However, it exerts strong shear stress on cells, producing damage, and clogging of membrane pores also reduces the efficiency of filtration. Another popular approach for target cell purification is the use of magnetic particles, called MACS (magnetic cell separation) ${ }^{7-12}$. In this method, target cells are recognized by their specific antibodies immobilized on the magnetic particle surfaces, and collected by magnetic field application. The magnetic particles are dispersed in solvent and attached to target cells; therefore, this method can avoid the shear stress damage to cells upon use of the filtration method; however, target cells should be recognized with probe molecules such as antibodies, and size-dependent cell separation has been difficult using the conventional MACS method.

Here, we propose a new method of size-selective target cell separation that avoids shear stress by using precisely size-controlled hemispherical superparamagnetic microparticles, "magcups".

\section{Results}

We have fabricated precisely size-controlled hemispherical superparamagnetic microparticles ("magcups") having three layers, namely, a 2-nm nickel layer inserted between 15-nm silicon dioxide layers by vapor deposition. Figure 1 (a) shows an overview of the fabrication of a magcup. The fabrication procedure is fundamentally based on a method described in previous reports ${ }^{13-18}$; specifically, in this study, we precisely controlled the thickness of nickel $(\mathrm{Ni})$ up to $2 \mathrm{~nm}$ and the spacing silicon dioxide $\left(\mathrm{SiO}_{2}\right)$ thickness larger than $15 \mathrm{~nm}$ to maintain the superparamagnetic conditions. For the magnetic layers, other elements such as iron or cobalt can be also used as suitably, and in this study, Ni was chosen because of its stability in solvents containing high concentration of salts like culture medium.

Firstly, 11 types of precisely size-controlled polystyrene spheres $(7,10,15,20,25,30,40,50,60,70$ or $80 \mu \mathrm{m}$ in diameter) were placed on a flat silicon substrate, and $\mathrm{Ni}$ was deposited on the spheres by thermal deposition with strict control of its thickness set to $2 \mathrm{~nm}$ to convey the property of superparamagnetism to the fabricated particles (see also Suppl. Movies S1 and S2). To enhance the magnetic charge of a magcup, we added more Ni layers on it (this time, finally three layers) with spacing material of $\mathrm{SiO}_{2}$, with strict control of its thickness at $15 \mathrm{~nm}$ (this 


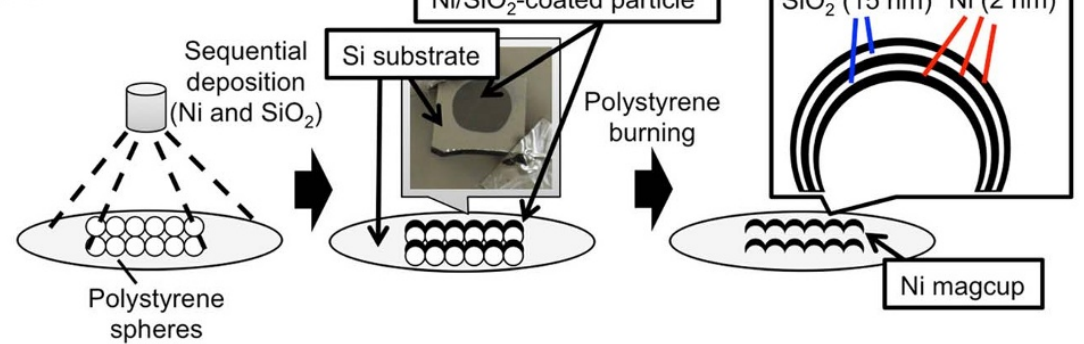

(b)

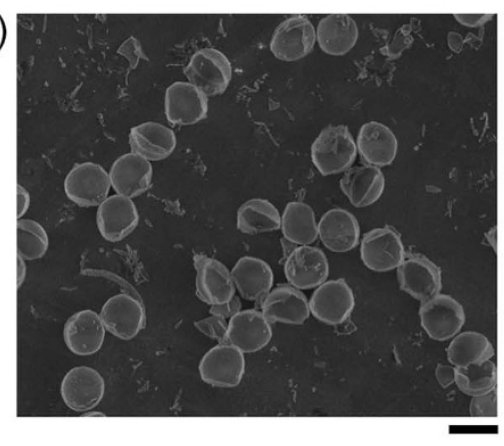

(c)

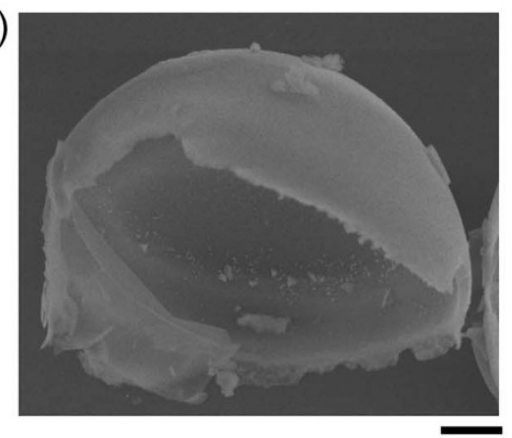

Figure 1 Fabrication of the magcup. (a) Schematic images of the fabrication. First, $\mathrm{Ni}$ and $\mathrm{SiO}_{2}$ were sequentially deposited on the polystyrene beads at thicknesses of 2 and $15 \mathrm{~nm} 3$ and 2 times, respectively. Next, the deposited polystyrene beads were burned in an electric furnace to remove polystyrene casts from the particles. Then, the magcups composed of $\mathrm{Ni}$ and $\mathrm{SiO}_{2}$ layers were fabricated. (b, c) FE-SEM images of fabricated magcups at low (b) and high (c) magnifications. Cup size, $10 \mu \mathrm{m}$. Bars are $10 \mu \mathrm{m}$ (b) and $1 \mu \mathrm{m}(\mathrm{c})$.

time, two layers between three layers of $\mathrm{Ni}$ ), because spacing of less than $15 \mathrm{~nm}$ is insufficient to maintain the superparamagnetic properties and the Ni layers change to exhibit ferromagnetic properties. Finally, the $\mathrm{Ni} / \mathrm{SiO}_{2}$-coated particles were heated at $500^{\circ} \mathrm{C}$ for $17 \mathrm{~h}$ to remove the polystyrene sphere cast; then, magcups were obtained (Figs. 1 (b) and (c)). We confirmed complete removal of polystyrene spheres through immobilizations of biomolecules onto interiors of magcups in our previous studies ${ }^{18,19}$, and also confirmed by energy dispersive X-ray spectrometry (EDS) of the FE-SEM analyses as significant decrease of carbon peak in the measurements (Suppl. Fig. S1).

Subsequently, we examined whether the hemispherical shape of a magcup can be applied for the size filtration of cells. First, sizeuniform polystyrene beads of $10 \mu \mathrm{m}$ in diameter $\left(2 \times 10^{7} / \mathrm{mL}\right)$ were pre-coated with bovine serum albumin to enhance binding affinity to the inner surface of the magcups; then, the polystyrene beads were mixed with magcups having an inner diameter of $7,10,15,20,25,30$, $40,50,60,70$ or $80 \mu \mathrm{m}\left(3 \times 10^{6} / \mathrm{mL}\right)$. After $1 \mathrm{~h}$ of incubation, the polystyrene beads were successfully captured in the cavity of the magcups as shown in Fig. 2 (a). The number of bead-conjugated magcups was counted for at least 200 cups for each diameter to evaluate the size-selective acquisition ability of the inner cavity of the magcups (Fig. 2 (b)). As shown in the graph, the beads were specifically captured by magcups with diameters equal to and larger than $15 \mu \mathrm{m}$, and the frequency of bead-conjugated magcups gradually increased with an increase of the size of the magcup, up to $90 \%$ at maximum; however, the magcups smaller than $15 \mu \mathrm{m}$, which is the same size or smaller than the polystyrene beads, did not capture any polystyrene beads $(0 \%$ for both $10-\mu \mathrm{m}(\mathrm{n}=320)$ and $7-\mu \mathrm{m}$ magcups $(\mathrm{n}=331))$. Similar results were acquired in the case of $7-\mu \mathrm{m}$ polystyrene beads ( $0 \%$ in $7-\mu \mathrm{m}$ magcups, and $21 \%$ in $10-\mu \mathrm{m}$ magcups) and $15-\mu \mathrm{m}$ polystyrene beads ( $0 \%$ in $15-\mu \mathrm{m}$ and smaller magcups). In addition, each suspension of two different size beads having 10 and $40 \mu \mathrm{m}$ (Fig. 2 (c)), 20 and $40 \mu \mathrm{m}$ (Fig. 2 (d)), or 30 and $40 \mu \mathrm{m}$ (Fig. 2 (e)) was mixed with magcups having an inner diameter of 20, 30,40 or $50 \mu \mathrm{m}$ to evaluate filtration abilities of magcups. In results, smaller beads than magcup diameters were only captured from the bead suspensions (e.g., $10 \mu \mathrm{m}$ beads were only collected using $20 \mu \mathrm{m}$ magcups from 10 and $40 \mu \mathrm{m}$ bead mixture in Fig. 2 (c)). These results indicate that the inner cavity of the magcup behaves as a size filter to exclude targets larger than the magcup diameter. An exact threshold of size filtration was achieved by uniform size distribution of magcups, with a low coefficient of variation (CV) of less than $3 \%$. For twice and more larger magcups than target beads, a portion of magcups captured more than two beads in a cup with an increase of the frequency of bead-conjugated magcups. For larger magcups than both bead diameters (i.e., $50 \mu \mathrm{m}$ magcups), large beads (i.e., $40 \mu \mathrm{m}$ beads) were more frequently captured than small one, might be caused on the depletion effect in which entropic driving forces to conjugate beads with magcups were acted to make a total overlapping volume between beads and magcups as maximum under co-existence of nanoscopic polymers, which yielded frequent acquisition of beads having close diameters with that of magcups ${ }^{20,21}$.

We also evaluated size-selective collection of the fabricated magcups for the specific target cells. A size of magcup (any one of the 11 types of magcup having a diameter from 7 to $80 \mu \mathrm{m}$ ) was mixed with human acute lymphoblastic leukemia cells (CCRF-CEM, $12 \pm$ $2 \mu \mathrm{m}$ in diameter) and incubated for $30 \mathrm{~min}$. Then, the magcups were collected by the application of a magnetic field using a magnet. As clearly shown in the scanning electron micrographs, target cells were successfully captured in the magcup cavities with sizes of $15 \mu \mathrm{m}$ (Fig. 3 (a)) and $20 \mu \mathrm{m}$ (Fig. 3 (b)). Almost all cells were specifically attached to the interior of magcups, because a contact area between the cell and a cup exterior is small and therefore, adhesion force is too weak for enough attachment of the cell to the cup exterior. The frequency of cell-conjugated magcups was determined by counting in the same manner as for the previous polystyrene bead collection to evaluate size-selective target filtration (Fig. 3 (c)). From the results, target cells were size-specifically captured by magcups with diameters equal to and larger than $15 \mu \mathrm{m}$, but were not captured by 7 - and $10-\mu \mathrm{m}$ magcups. Diameters of captured cells using $15 \mu \mathrm{m}$ magcups were minutely measured $(n=200)$ and the distribution was compared with that before the collection assays $(n=400)$. Figure $3(d)$ shows the result of com- 
(a)

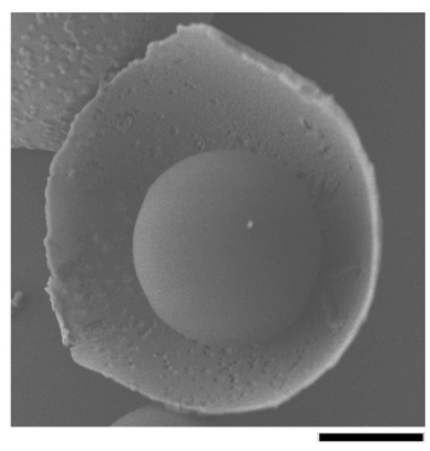

(c)

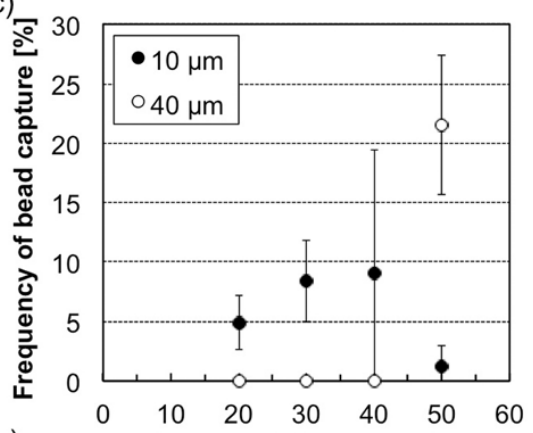

(e)

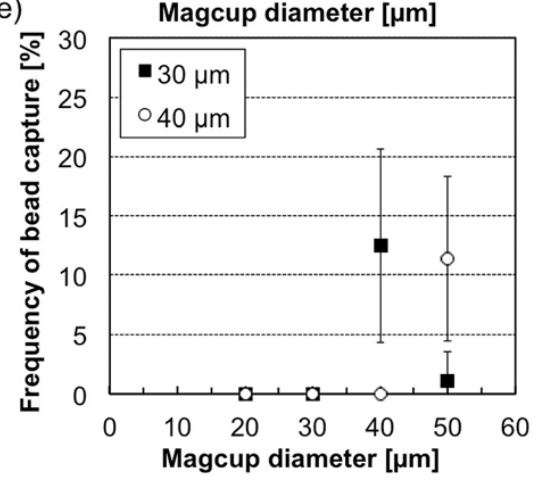

(b)

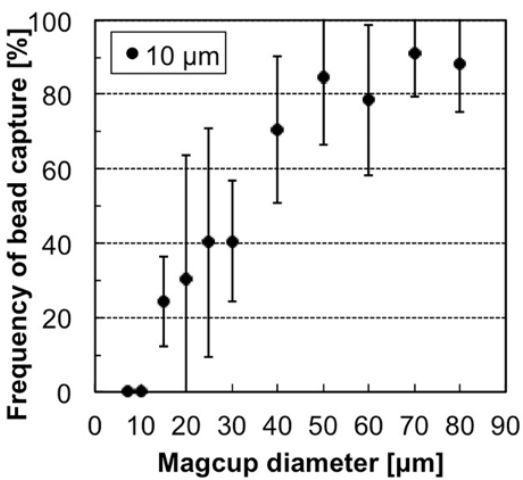

(d)

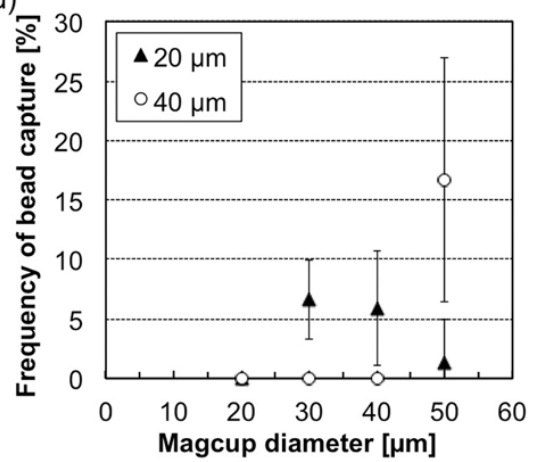

Figure $2 \mid$ Capture of model target beads using the magcups. (a) An FE-SEM image of a 20- $\mu \mathrm{m}$ magcup in which a $10-\mu \mathrm{m}$ bead was captured. Bar, $5 \mu \mathrm{m}$. (b) Relationship between the frequency of 10- $\mu \mathrm{m}$-bead-conjugated magcups and the cup diameter. (c-e) Relationship between the frequency of 10- or 40- (c), 20- or 40- (d), and 30- or 40- $\mu \mathrm{m}$ (e) bead-conjugated magcups and the cup diameter. Error bars indicate standard deviations (S.D.).

parison, and as shown in the figure, cells smaller than $15 \mu \mathrm{m}$ were only purified by the magcup collection assays. These results are consistent with that in the previous polystyrene bead collection; namely, the magcups only collect target cells that are smaller than their inner cavity.

After the collection of target cells using the magcups, the survival of collected cells was evaluated. Collected cells trapped in the magcups were placed into small incubation chambers made of poly(dimethylsiloxane) (PDMS) on a cover slip to prevent their diffusion, and the survival was evaluated using two-color fluorescent staining of live (Calcein AM, green) and dead cells (EthD-III, red). Figure 4 (a) shows optical and fluorescent microscope images of the staining results for six typical samples (S1-S6). As shown in the figure, cells captured by the magcups (indicated as " $\mathrm{m}+\mathrm{c}$ ") were stained either green (live) or red (dead), but empty magcups (indicated as " $m$ ") were not stained for both colors. The survival rate was $87 \%$, which is almost the same as that before the collection assay, $93 \%$, indicating that the damage associated with the collection assay was almost negligible. Next, collected cells were incubated in cell culture medium with time-lapse observation. Figure 4 (b) shows typical sequential optical bright field micrographs of the time-lapse observation taken every $10 \mathrm{~min}$ (the movie is also available online as Suppl. Movie S3).
The cell, which might have been ready to enter the mitosis phase, first escaped from its magcup $80 \mathrm{~min}$ after the start of the observation, and $120 \mathrm{~min}$ after the start, it divided into two cells. After incubation of collected cells with magcups, cell survival was evaluated again. Figure 4 (c) shows optical and fluorescent microscope images of the staining results by the same procedure as in Fig. 4 (a) after 5 days of incubation of collected cells. As shown in the pictures, collected cells survived even though they were incubated with the magcups. The survival rate was $91 \%$, which also indicated almost negligible damage associated with collection assays using magcups. The growth curves of collected cells were also determined at a bulk scale to evaluate their level of damage. In this evaluation, the numbers of cells were normalized by the number upon initial collection to avoid bias due to differences in collected cell number in each assay. Figure 4 (d) shows the results of cell increase for three independent collection assays with a curve of increase for the cultivated CCRF-CEM cell line. As shown in the figure, gradients of cell increases were almost the same as that of the cultured cell line, approximately doubling each day. These results clearly indicate that collected cells survived without serious damage, detached from the inner cavity of the magcup without any treatment, and could be re-cultivated in culture medium. 
(a)

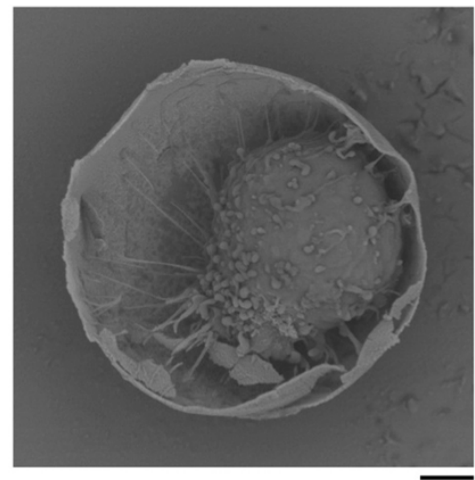

(c)

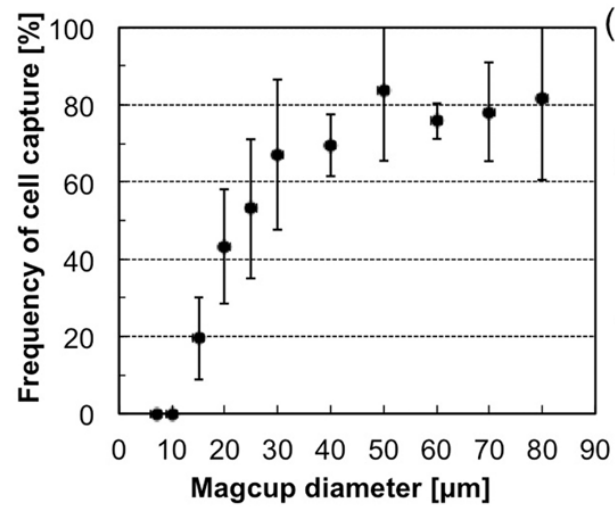

(b)
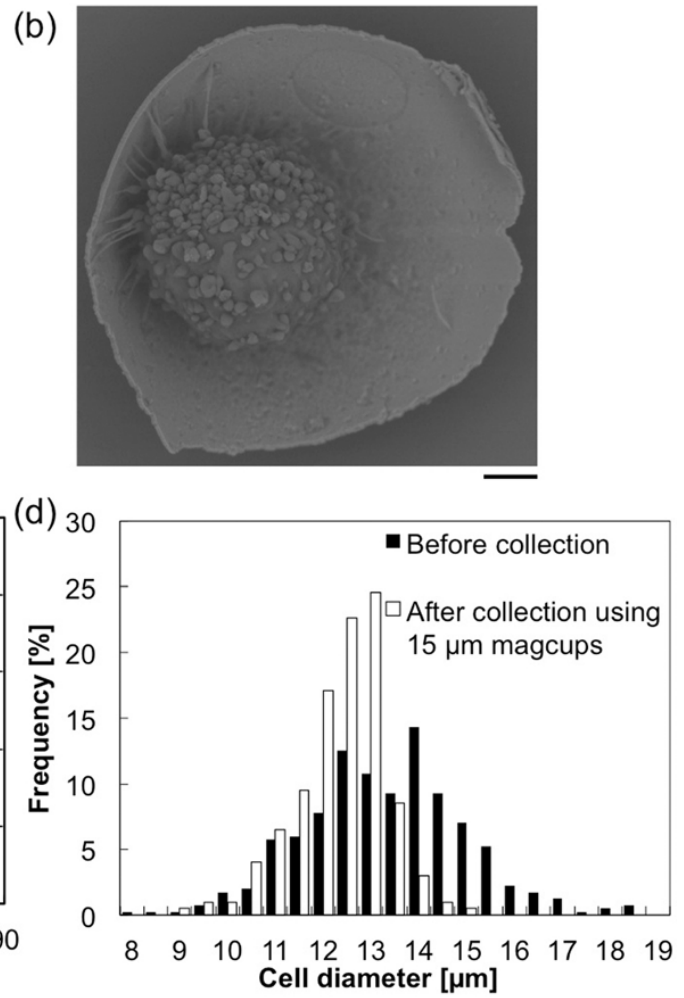

Figure 3 Capture of CCRF-CEM cells using the magcups. (a, b) Typical FE-SEM images of 15- (a) and 20- $\mu$ m magcups (b) in which the target cells were captured. Bar, $2 \mu \mathrm{m}$. (c) Relationship between the frequency of the target-cell-conjugated magcups and the cup diameter. (d) Distributions of cell diameters before and after cell collection assays using $15 \mu \mathrm{m}$ magcups. Error bars indicate S.D.

\section{Discussion}

In this study, precisely size-controlled magcups were fabricated and used to collect desired target cells depending on their sizes as a physical shape biomarker. We compared the developed magcup method with popular conventional methods, e.g., MACS and membrane filtration. There are five important viewpoints for cell purification, i.e., cell size filtration, molecular biomarker-based cell collection, combined purification of size and molecular biomarker, batch-treatment (or high-throughput treatment), and non-invasive (damage-free) cell collection, and the magcup method can cover all these five characteristics. As MACS is one of the most popular and powerful methods for expressed target molecule-based cell purification, that all cells on which target biomarker molecules are expressed are collected with recognition and target-antibody interaction by antibodies immobilized on the magnetic particles regardless of their sizes. Therefore, this method lacks the ability of size-filtration function. In contrast, the membrane filtration can provide size filtration by controlling pore size on the membrane. However, strong shear stress which is applied to cells during the filtration sometimes gives serious damage to cells, or it might give some clotting of cells on the membrane surface ${ }^{22-24}$. Upon comparison of these methods, we can regard our method as an advanced MACS method which can cover size filtration function with maintaining all advantages of conventional MACS method, and our method can overcome all existing problems in conventional membrane filtration method. In addition, our method can combine molecular filtration with size filtration at the cell purification. Moreover, the addition of size filtration function to the MACS method can give a potential to achieve more reliable purification of target cells by avoiding collection of clustered cells, in which undesired cells are contaminated.

In this study, magcups were composed of stacked $\mathrm{Ni}$ and $\mathrm{SiO}_{2}$ layers to add superparamagnetic properties for the cups. There are some indications about the toxicity of $\mathrm{Ni}$ for living systems based on in vitro ${ }^{25}$ and in vivo ${ }^{26}$ examinations. For studies in vitro, incorporation of Ni compounds into the cell could be harmful in terms of the maintenance of normal functions of the living system; therefore, dissolution of $\mathrm{Ni}$ compounds should be taken into account. $\mathrm{Ni}$ is in general water-insoluble and, in fact, the magcups used in this study retained their shape completely even though they were exposed to solvents in order to perform cell collection assays. Moreover, collected cells survived even when they were cultivated with magcups for a long period, as shown in Fig. 4, indicating low toxicity of the magcups for target cells in the in vitro assays.

In conclusion, various sizes of fabricated superparamagnetic magcup can be used as a tool for cell filtration and collection, which is as convenient as conventional MACS, adding the function of cell-size filtration, which is a major advantage of the magcups as a useful target cell collection tool.

\section{Methods}

Fabrication of cup-shaped superparamagnetic hemispheres. The superparamagnetic hemispheres (referred to as "magcups" in the text, and also hereafter) were fabricated using polystyrene spheres as templates by the following procedures $^{13-18}$. First, commercially available suspensions of polystyrene spheres of 7 to $80 \mu \mathrm{m}$ in diameter (DYNOSPHERES; nominal diameters, 7.088, 10.14, 15.62, 20.31 and $25.01 \mu \mathrm{m}$; coefficients of variation $(\mathrm{CV}), 0.80,1.20,1.17,1.17$ and $2.98 \%$; JSR, Japan; and Duke Standards, nominal diameters, 29.75, 39.94, 50.2, 59.2, 69.1 and $79.0 \mu \mathrm{m}$, and CV, 1.4, 1.3, 1.0, 1.4, 1.2 and 1.3\%, respectively; Thermo Scientific, CA, USA) were dropped onto clean flat silicon ( $\mathrm{Si}$ ) substrates as one diameter of the sphere on a substrate, and dried. Next, the polystyrene spheres were placed into a vacuum evaporator (VPC-1100, ULVAC, Japan) to coat elements on their surfaces, and nickel $(\mathrm{Ni})$ and silicon dioxide $\left(\mathrm{SiO}_{2}\right)$ were sequentially coated on the spheres alternately and as thin enough to convey superparamagnetism, typically $2 \mathrm{~nm}$ for Ni and $15 \mathrm{~nm}$ for $\mathrm{SiO}_{2}$. Finally, the element-coated spheres were placed into an electric furnace (MMF-2, Asone, Japan) and incubated at $500^{\circ} \mathrm{C}$ for $17 \mathrm{~h}$ to remove the polystyrene sphere templates. To use the obtained magcups in dispersed states, a suitable solvent, typically ultra-pure water containing $0.1 \%$ Tween 20 , was dropped on a Si substrate attached to the magcup, and the cup was dislodged from the substrate by the gentle application of ultrasound in a sonicator; then, the cup was dispersed in the dropped solvent. 


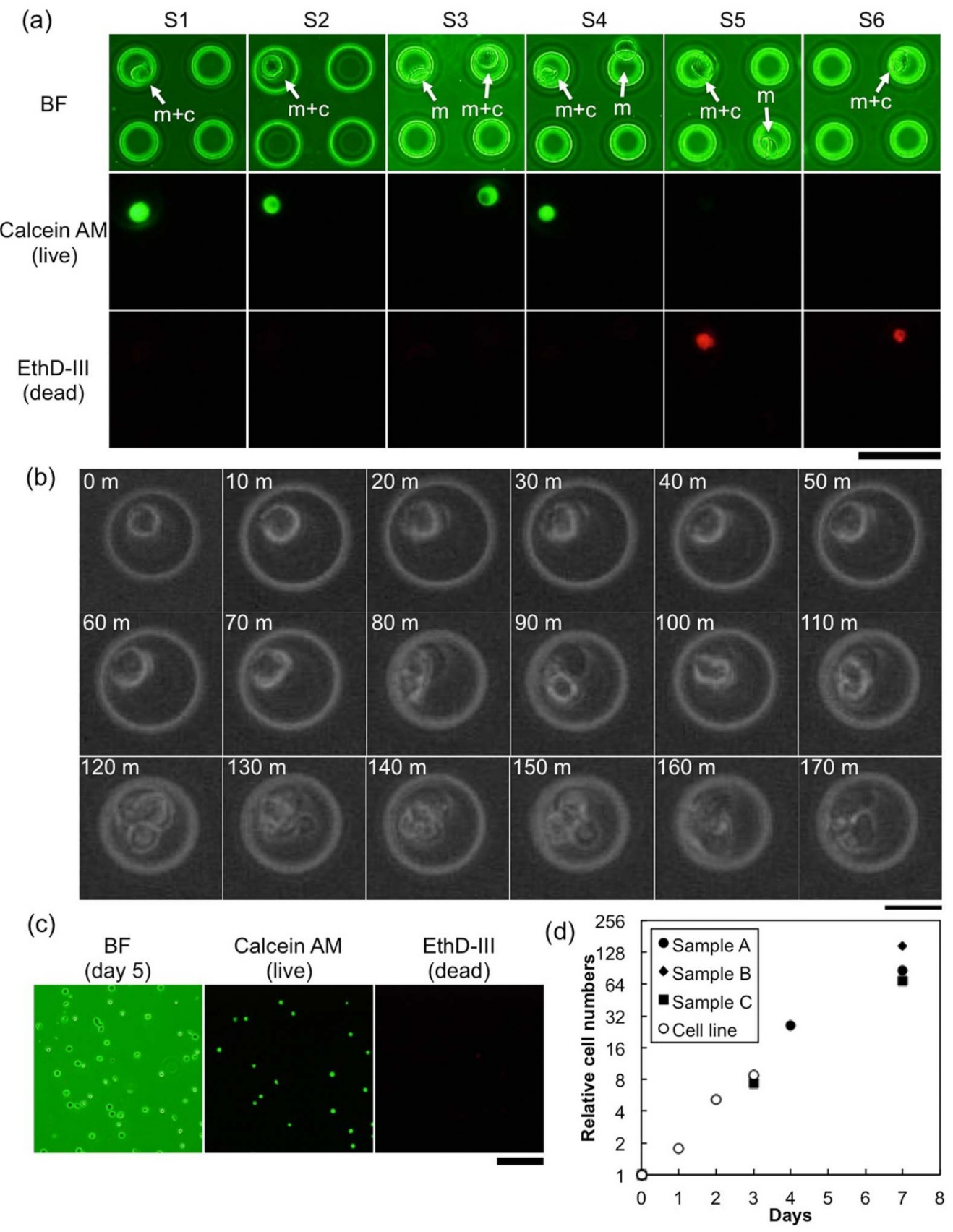

Figure $4 \mid$ Evaluation of collected cell survival. (a) Optical and fluorescent microscope images of two-color fluorescent staining results for six typical samples (S1-S6). Cells captured by the magcups $(\mathrm{m}+\mathrm{c})$ and empty magcups $(\mathrm{m})$ are indicated by arrows in bright field (BF) images. Bar, $50 \mu \mathrm{m}$. (b) Time-lapse observation of a collected cell. The cell still in the magcup was placed into a small PDMS chamber (shown as an outer white circle in the pictures) to prevent diffusion, and pictures were taken every $10 \mathrm{~min}$. The time elapsed since the start of monitoring is shown in each picture. Bar, $20 \mu \mathrm{m}$. (c) Optical and fluorescent microscope images of two-color fluorescent staining results for collected cells using magcups after 5 days of incubation in culture medium. Bar, $100 \mu \mathrm{m}$. (d) Relationships between relative cell numbers and the periods elapsed since the start of cultivation for three independent cell collection assays by the magcup (black circle, diamond, and square). Results for a cultivated CCRF-CEM cell line are also plotted (white circle).

Collection of polystyrene beads using the magcups. For the bead collection using the magcups, bovine serum albumin (BSA)-coated fluorescent polystyrene beads (Fluoresbrite $^{\mathrm{TM}}$ carboxylate YG microspheres, nominal diameter of $10.08 \pm 0.97 \mu \mathrm{m}$; Polyscience, USA) were used as a model target. The BSA molecules were coated to allow non-specific binding of the beads to the magcup surfaces. For the immobilization of BSA onto the bead surface, $2 \times 10^{6}$ carboxylated beads were activated with $10 \mathrm{mg} / \mathrm{mL}$ 1-ethyl-3 (3-dimethylaminopropyl) carbodiimide hydrochloride (EDC, Thermo Scientific, USA) and $10 \mathrm{mg} / \mathrm{mL}$

N-hydroxysulfosuccinimide (NHS, Wako, Japan) in $\mathrm{pH} 5.0$

2-morpholinoethanesulfonic acid (MES) for $15 \mathrm{~min}$ at room temperature, washed with MES 3 times, reacted with 1\% BSA (Life Technologies, USA) in pH 7.4

phosphate-buffered saline (PBS) for $1 \mathrm{~h}$ at room temperature, and washed with PBS 3 times. Then, $3 \times 10^{5}$ magcups of various diameters (i.e., $7,10,15,20,25,30,40,50,60$, 70 or $80 \mu \mathrm{m}$ ) were reacted with the BSA-coated beads in ultra-pure water containing
$0.1 \%$ Tween 20 for $1 \mathrm{~h}$ at room temperature. After the reaction, the sample was washed with the same kind of solvent 3 times and observed using a fluorescent microscope to count the number of bead-conjugated magcups.

For the collection of two different size beads, polystyrene beads (diameters, 10.14, 20.31, 29.75 and $39.94 \mu \mathrm{m}, \mathrm{CV}, 1.20,1.17,1.4$ and 1.3\%, respectively, JSR and Duke) were mixed with $1 \% \mathrm{BSA}$ in $\mathrm{pH} 7.4 \mathrm{PBS}$ for $1 \mathrm{~h}$ at room temperature, washed with PBS 3 times, and adjusted as two bead volumes as the same in the solvent at $0.1 \%(\mathrm{v} / \mathrm{v})$ concentrations. The suspensions were reacted with $20,30,40$ or $50 \mu \mathrm{m}$ magcups as the same procedure with the above single size bead collection assays.

Collection of target cells using the magcups. For the collection of target cells using the magcups, a human acute lymphoblastic leukemia cell line (CCRF-CEM) was chosen as a model target, and was maintained in culture medium at $37^{\circ} \mathrm{C}$ and under $5 \% \mathrm{CO}_{2}$ in RPMI 1640 (Life Technologies) supplemented with $10 \%$ heat-inactivated 
fetal bovine serum (Asahi Glass, Japan) and $100 \mathrm{U} / \mathrm{mL}$ penicillin- $100 \mu \mathrm{g} / \mathrm{mL}$ streptomycin (Life Technologies). In the reaction, $1 \times 10^{6}$ cells were reacted with $3 \times$ $10^{5}$ magcups in binding buffer ${ }^{27}$, consisting of Dulbecco's PBS containing $25 \mathrm{mM}$ glucose, $5 \mathrm{mM} \mathrm{MgCl}_{2}$, and $1 \% \mathrm{BSA}$, for $30 \mathrm{~min}$ at room temperature with gentle mixing every $10 \mathrm{~min}$. After the reaction, the sample was washed with binding buffer 3 times, dropped on a glass, and observed using an optical microscope to count the number of cell-conjugated magcups. For the measurement of collected cell diameters, the optical microscope images were taken and the diameters were calculated using image analysis software (Image J 1.48, http://imagej.nih.gov/ij/).

Re-cultivation of target cells collected with the magcups. To evaluate the survival of the cells collected using the magcups, time-lapse observation of the cells was performed. Firstly, a small poly(dimethylsiloxane) (PDMS) chamber array was fabricated in a cell culture dish as follows. The PDMS (SYLGARD 184 silicon elastomer, Dow Corning, USA) sol was dropped onto a Si mold on which microcolumns ( $30 \mu \mathrm{m}$ in diameter and $30 \mu \mathrm{m}$ in height) had been pre-fabricated. The dropped PDMS sol was heated at $90^{\circ} \mathrm{C}$ for $1 \mathrm{~h}$ to harden it. After heating, the PDMS was then peeled off from the mold and attached on a glass-based cell culture dish (Asahi Glass).

The cells collected using the magcups were placed into the PDMS chamber while still captured in the magcup and a few milliliters of cell culture medium was carefully added to the dish. The dish was set on an inverted microscope (IX-71, Olympus, Japan) combined with both a stage top incubator (Tokai Hit, Japan) and a time-lapse CCD acquisition system. Images of the re-cultivated cells were then taken every $10 \mathrm{~min}$.

For two-color fluorescent staining of live and dead cells, a commercially available live/dead cell staining kit (PromoCell, Germany) was used. The kit contained two fluorescent probes, Calcein AM (live cell staining in green) and EthD-III (dead cell staining in red), and those probes were adjusted to $10 \mu \mathrm{M}$ using PBS. The probe solution was applied to the collected cells still captured in the magcups, and incubated for $30 \mathrm{~min}$ at room temperature. After the incubation, the cells were washed with PBS and microscopic observations were performed.

FE-SEM observation. The results of the magcup reactions were confirmed by direct observation of the cup using field emission scanning electron microscopy (FE-SEM; JSM-6701F, JEOL, Japan) with a backscattered electron (BSE) detector (SM-74071, JEOL). In the case of cell-conjugated magcup observation by FE-SEM, the reacted sample was immersed in 70, 80, 90, 95 and $99 \%$ ethanol sequentially for dehydration. The cells were additionally immersed in $100 \%$ ethanol, which was dehydrated with copper sulfate, twice, and then tert-butyl alcohol, and incubated at $-20^{\circ} \mathrm{C}$ for $1 \mathrm{~h}$. The specimen was placed in a freeze-dry system (Labconco, USA) and freeze-drying was performed. Before the FE-SEM observation, samples were coated with platinum using an automatic fine coater (JFC-1600, JEOL) with a sputtering current of $30 \mathrm{~mA}$ for $40 \mathrm{~s}$. The observation conditions were as follows: $5 \mathrm{kV}$ acceleration voltage, $\times 1,000$ to $\times 5,000$ magnification, $200 \mathrm{pA}$ probe current, $8 \mathrm{~mm}$ working distance, $20 \mathrm{~s}$ capture time, $1280 \times 1024$ pixels in a picture, and secondary electron (SE) and BSE detection mode. An incident electron beam was vertically applied to the sample surface, and the BSE was monitored with its scattering angle ranging between $16^{\circ}$ and $60^{\circ}$ from the axis of the incident electron beam.

1. Rostagno, P., Moll, J. L., Bisconte, J. C. \& Caldani, C. Detection of rare circulating breast cancer cells by filtration cytometry and identification by DNA content: sensitivity in an experimental model. Anticancer Res. 17, 2481-2485 (1997).

2. Vona, G. et al. Isolation by size of epithelial tumor cells: a new method for the immunomorphological and molecular characterization of circulatingtumor cells. Am. J. Pathol. 156, 57-63 (2000).

3. Hosokawa, M. et al. Size-selective microcavity array for rapid and efficient detection of circulating tumor cells. Anal. Chem. 82, 6629-6635 (2010).

4. Hosokawa, M. et al. Size-based isolation of circulating tumor cells in lung cancer patients using a microcavity array system. PLOS ONE 8, e67466 (2013).

5. Davis, J. A. et al. Deterministic hydrodynamics: taking blood apart. Proc. Natl. Acad. Sci. USA 103, 14779-14784 (2006).

6. Desitter, I. et al. A New Device for Rapid Isolation by Size and Characterization of Rare Circulating Tumor Cells. Anticancer Res. 31, 427-441 (2011)

7. Dunlop, E. H., Feiler, W. A. \& Mattione, M. J. Magnetic separation in biotechnology. Biotechnol. Adv. 2, 63-74 (1984).

8. Owen, C. S. \& Sykes, N. L. Magnetic labeling and cell sorting. J. Immunol. Methods 73, 41-48 (1984)

9. Kronick, P. \& Gilpin, R. W. Use of superparamagnetic particles for isolation of cells. J. Biochem. Biophys. Methods 12, 73-80 (1986).

10. Lea, T. et al. Monosized, magnetic polymer particles: their use in separation of cells and subcellular components, and in the study of lymphocyte function in vitro. J. Mol. Recognit. 1, 9-18 (1988).

11. Wang, E. C., Borysiewicz, L. K. \& Weetman, A. P. Cell sorting using immunomagnetic beads. Methods Mol. Biol. 10, 347-357 (1992).
12. Karumanchi, R. S., Doddamane, S. N., Sampangi, C. \& Todd, P. W. Field-assisted extraction of cells, particles and macromolecules. Trends Biotechnol. 20, 72-78 (2002).

13. Takei, H. \& Shimizu, N. Gradient sensitive microscopic probes prepared by gold evaporation and chemisorption on latex spheres. Langmuir 13, 1865-1868 (1997).

14. Takei, H. Surface-adsorbed polystyrene spheres as a template for nanosized metal particle formation: Optical properties of nanosized Au particle. J. Vac. Sci. Tech. B 17, 1906-1911 (1999)

15. Himmelhaus, M. \& Takei, H. Cap-shaped gold nanoparticles for an optical biosensor. Sens. Actuators B: Chem. 63, 24-30 (2000).

16. Kim, H., Yasuda, K. \& Takei, H. Production of nanoscopic metal labels for electron microscopy: Specific detection of target DNA. Sens. Actuators B: Chem. 142, 1-6 (2009).

17. Kim, H., Takei, H. \& Yasuda, K. Production of Size-Controlled Nanoscopic CapShaped Metal Shells. Jpn. J. Appl. Phys. 49, 048004 (2010).

18. Kim, H., Hayashi, M., Terazono, H., Takei, H. \& Yasuda, K. Production of DoubleLayered Metal Nanocups for Artificial Nanospace of Biomolecular Reaction. Jpn J. Appl. Phys. 50, 06GJ03 (2011).

19. Kim, H., Terazono, H., Takei, H. \& Yasuda, K. DNA hybridization efficiency on concave surface nano-structure in hemispherical Janus nanocups. Langmuir 30, 1272-1280 (2014).

20. Asakura, S. \& Oosawa, F. On Interaction between 2 Bodies Immersed in a Solution of Macromolecules. J. Chem. Phys. 22, 1255-1256 (1954).

21. Sacanna, S., Irvine, W. T. M., Chaikin, P. M. \& Pine, D. J. Lock and key colloids. Nature 464, 575-578 (2010).

22. Lin, H. K. et al. Portable filter-based microdevice for detection and characterization of circulating tumor cells. Clin. Cancer Res. 16, 5011-5018 (2010).

23. Zheng, S. et al. 3D microfilter device for viable circulating tumor cell (CTC) enrichment from blood. Biomed. Microdev. 13, 203-213 (2011).

24. Harouaka, R. A. et al. Flexible micro spring array device for high-throughput enrichment of viable circulating tumor cells. Clin. Chem. 60, 323-333 (2014).

25. Chromium, nickel and welding. IARC Monogr. Eval. Carcinog. Risks Hum. 49, $1-648$ (1990).

26. Report of the International Committee on Nickel Carcinogenesis in Man. Scand. J. Work Environ. Health 16, 1-82 (1990).

27. Terazono, H., Anzai, Y., Soloviev, M. \& Yasuda, K. Labelling of live cells using fluorescent aptamers: binding reversal with DNA nucleases. J. Nanobiotechnology 8, 8 (2010).

\section{Acknowledgments}

We gratefully thank Ms. M. Murakami and Ms. M. Naganuma for their technical assistance. We also thank JEOL Ltd. for the assistance in obtaining EDS spectra.

\section{Author contributions}

H.K. designed experimental conditions throughout the study, and performed measurements and data analysis. H. Terazono contributed to determine experimental conditions for cell capture, time-lapse observation and re-cultivation of collected cells, and data processing throughout the study. H. Takei conceived the fabrication method of Mag cups and conducted the particle fabrication. K.Y. conceived and conducted the design of this study. The manuscript was written by H.K. and K.Y.

\section{Additional information}

Supplementary information accompanies this paper at http://www.nature.com/ scientificreports

Competing financial interests: Yes, there is potential Competing Interest. The funding was supported by the Japan Prize Foundation research grants, JSPS KAKENHI grant number 24681025 and Kanagawa Prefector's local basic science funding for On-chip Cellomics Project in Kanagawa Academy of Science and Technology. Funders had no role in study design, data collection and analysis, decision to publish, or preparation of the manuscript. How to cite this article: Kim, H., Terazono, H., Takei, H. \& Yasuda, K. Cup-Shaped Superparamagnetic Hemispheres for Size-Selective Cell Filtration. Sci. Rep. 4, 6362; DOI:10.1038/srep06362 (2014).

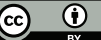

This work is licensed under a Creative Commons Attribution 4.0 Internation License. The images or other third party material in this article are included in the article's Creative Commons license, unless indicated otherwise in the credit line; if the material is not included under the Creative Commons license, users will need to obtain permission from the license holder in order to reproduce the material. To view a copy of this license, visit http://creativecommons.org/licenses/by/4.0/ 